\title{
Average sampling in mixed shift-invariant subspaces with generators in hybrid-norm spaces
}

\author{
Haizhen $\mathrm{Li}^{1}$ and Yan Tang ${ }^{1}$ \\ ${ }^{1}$ Guilin University of Electronic Technology
}

April 26, 2021

\begin{abstract}
This paper mainly studies the average sampling and reconstruction in shift-invariant subspaces of mixed Lebesgue spaces $\$ \mathrm{~L}^{\wedge}\{\mathrm{p}, \mathrm{q}\}\left(\backslash \operatorname{mathbb}\{\mathrm{R}\}^{\wedge}\{\mathrm{d}+1\}\right) \$$, under the condition that the generator $\$ \backslash$ varphi $\$$ of the shift-invariant subspace belongs to a hybrid-norm space of mixed form, which is weaker than the usual assumption of Wiener amalgam space and allows to control the orders $\$ \mathrm{p}, \mathrm{q} \$$. First, the sampling stability for two kinds of average sampling functionals are established. Then, we give the corresponding iterative approximation projection algorithms with exponential convergence for recovering the time-varying shift-invariant signals from the average samples.
\end{abstract}

\section{Hosted file}

manuscript.pdf available at https://authorea.com/users/410060/articles/519552-averagesampling-in-mixed-shift-invariant-subspaces-with-generators-in-hybrid-norm-spaces 